\title{
Peer Consultation: An Enriching Necessity Rather Than a Luxury for Psychologists During and Beyond the Pandemic
}

\author{
Adriana S. Miu · Anitha Joseph · Ellie Hakim · Erin D. Cox · Ellen Greenwald
}

Published online: 6 January 2022

(c) National Register of Health Service Psychologists 2022

\begin{abstract}
The pandemic increased professional and personal demands on psychologists, resulting in higher levels of burnout and clinician isolation (Aafjes-van Doorn et al., 2020; Shklarski et al., 2021). Meanwhile, professional consultation is less available due to remote work and social distancing (Sasangohar et al., 2020). Without adequate consultation, psychologists' burnout and isolation may impair their ability to provide quality clinical care (Maslach \& Leiter, 2016). Limited literature focuses on how to support psychologists during this unprecedented time. We discuss assessment tools to identify needs for peer consultation and effective consultation group strategies. Our case example of a peer consultation group demonstrates how peer consultation can support psychologists and mitigate the unique challenges throughout the pandemic.
\end{abstract}

Keywords Peer consultation · Burnout $\cdot$ Pandemic

\section{Clinical Vignette}

Jane is an early career clinical psychologist at an academic medical center, where she primarily provides therapy to patients with trauma and depression at a busy adult outpatient clinic. She provides many back-to-back therapy sessions every day. After six months at her new job, the pandemic hit and the clinic transitioned to a hybrid work schedule (i.e., partly remote and partly in-clinic). Suddenly, she had to learn to conduct teletherapy with a challenging clinical population. Although some patients embraced teletherapy, others were difficult to engage and were frequently distracted during sessions. Jane also recognized that the pandemic caused significant social isolation and worsened symptoms for her patients. Jane did not see much progress in therapy for some of her patients and subsequently experienced self-doubt.

She wanted to consult with a peer, but not many providers were available when she was at the clinic because of the clinic's social distancing policy. When working remotely, colleagues' statuses often indicated "Busy" or "Not available." She felt isolated as if she were in private practice. She also knew that her peers were busy navigating the additional responsibilities of schooling their children while keeping a full-time clinical load. Meanwhile, Jane was very busy taking care of her children and often emotionally and physically drained after seeing so many patients. She knew subconsciously she should seek consultation on the cases that lacked progress, but she kept telling herself, "Well, maybe I just need to try longer. It's not really that urgent to take up somebody else's time."

Although there were clinic meetings every few weeks, these meetings focused on the transition to telehealth, case presentations, and occasional updates with other providers about mutual patients. She also did not want to take up valuable clinic meeting time for non-urgent cases. Jane feared being judged as unprofessional or incompetent if she were to discuss her burnout and challenging cases. With her children at home, Jane found herself caring for their physical and emotional needs between appointments. She had neither the time nor accessible colleagues for peer consultation, let alone for self-care. With the heavy caseload in addition to her family responsibilities, Jane was in survival mode just trying to get through the next 24 hours.

\section{Background}

\section{Peer Consultation Before the Pandemic}

Peer consultation was recommended and ethically necessary long before the pandemic (American Psychological Association, 2002). The American Psychological Association (APA) ethical guidelines require psychologists to 
obtain professional consultation when personal problems may interfere with professional duties (APA, 2002, Standard 2.06). Consultation relationships tend to be egalitarian and nonevaluative compared to supervisory relationships where the supervisor holds legal responsibility and oversees the supervisee's patients (Carney \& Jefferson, 2014). Consultation can be sought out with an expert in a specialty area or informally among peers as clinical dilemmas arise. Peer consultation groups are frequently described as small (4-6 members), informal, leaderless, and democratic. These groups tend to form through personal contact, hold regularly scheduled meetings, and follow a spontaneous format that blends case-centered and process-centered approaches (Napan, 2021; Carney \& Jefferson, 2014).

A key advantage of peer consultation groups is their potential to promote clinician effectiveness and ultimately protect the welfare of patients, which is an ethical obligation (APA, 2002). Psychologists in peer consultation express the need for regular consultation in order to receive guidance on problematic cases, discuss ethical and professional issues, and process countertransference concerns that arise with patients (Carney \& Jefferson, 2014). Extant literature indicates that peer consultation counters isolation and burnout by promoting a sense of community, collegiality, and connection (Carney \& Jefferson, 2014; Counselman \& Weber, 2004; Isobel et al., 2014). Starting a peer consultation group was linked to perceived improvement in clinical skills, personal well-being, and connection to a professional network among therapists who worked in rural, secluded areas (Isobel et al., 2014).

\section{Underutilization of Peer Consultation and Ethical Concerns}

Despite the ethical guidelines and benefits of peer consultation, consultation is underused and under-researched among psychologists. There is only one study that examined the utilization of peer consultation groups (Lewis et al., 1988). In a national survey of psychologists, only about one quarter of psychologists in private practice belonged to a peer consultation group, even though a majority of the sample (61\%) expressed interest in joining one (Lewis et al., 1988). Common barriers to peer consultation include practice settings that may not allot time for consultation, limited access to consultees, and ethical uncertainties related to confidentiality (Carney \& Jefferson, 2014). As a result, psychologists may rely on brief informal peer consultation or case management team meetings in outpatient settings, which may not allow enough time, reflection, or focused attention on the psychologists' needs and clinical difficulties. Without dedicated time and space for consultation, psychologists may not adequately address the intersection of the professional and personal issues that contribute to burnout, ethical questions, and clinical countertransference.

Furthermore, psychologists may lack self-awareness for the need of consultation. Vulnerable to cognitive biases, psychologists tend to rely on personal clinical experience versus evidence-based research or collegial advice in treatment decisions (Stewart \& Chambless, 2008). For instance, psychologists in independent practice reported a median of 12 sessions with a non-progressing patient before considering consultation (Stewart \& Chambless, 2008). Peer consultation groups can provide opportunities to increase awareness of biases, engage in critical thinking, and reduce shame and isolation that often arises in clinical work.

\section{Clinical and Ethical Challenges}

\section{Increased Clinical Demands and Burnout During the Pandemic}

There is an increased need for peer consultation as the pandemic has intensified the burden on psychologists. They carry heavier caseloads to meet the demands of a more distressed population (Sammons et al., 2021). Since the start of the pandemic, the rate of anxiety or depressive disorders rose approximately 30\% (Panchal et al., 2021). Psychologists take on more new patients and provide a greater frequency of appointments to their average patient (Slone et al., 2021). This is even more pronounced for patients with serious mental illness (Miu et al., 2020). Teletherapy has made treatment more accessible and reduced cancellation and no-show rates, which has led to busier schedules for psychologists. Within one of our clinical settings, for example, the number of appointments over the 2020-2021 year increased by 58\% compared to the 2018-2019 year, and the percentage of new patients seeking therapy increased by $20 \%$. Psychologists reported increases in patients' suicidal ideation or behavior, indicating greater symptom acuity in their clinical load (Sammons et al., 2021). In a survey of therapists during the pandemic, about two-thirds of therapists reported more fatigue and vicarious trauma than before the pandemic, which suggests greater burnout (Aafjes-van Doorn et al., 2020).

\section{Navigation of Telehealth Treatment and Associated Ethical Issues}

Additionally, psychologists had to navigate and adjust to teletherapy versus in-person sessions. Learning new platforms and addressing technological challenges presents an additional burden (Shklarski et al., 2021). Teletherapy adoption introduced new ethical and clinical concerns, as 
described in the clinical vignette. First, psychologists may not be able to ensure privacy, confidentiality, and security if patients conduct the sessions in settings where they could be overheard (Stoll et al., 2020). Second, psychologists need adequate training in teletherapy to ensure treatment fidelity, given that in-person therapeutic skills are not automatically translatable to telehealth therapeutic skills (Stoll et al., 2020). For many psychologists, the initial process of conducting teletherapy requires more energy to be fully present and connect with clients (Shklarski et al., 2021). Lastly, there are risk management issues when a patient is a danger to self or others but the provider is in a different physical location, which presents additional crisis management difficulties in a virtual format (Stoll et al., 2020). These ethical concerns are important to consider, but they do not negate telehealth benefits of increasing access to care and utilization (Sammons et al., 2021).

\section{Interference With Clinical Care Due to Personal Stressors}

Psychologists also face pandemic stressors shared by the general population (Shklarski et al., 2021). With lockdown and remote schooling, many psychologists navigated multiple roles and responsibilities including childcare and elder care, teaching support to school-aged children, and continued full-time clinical work. Psychologists who provide in-person treatment may fear contracting COVID-19 and inadvertently infecting their family and friends (Ledesma $\&$ Fernandez, 2021). Like the general population, psychologists may experience personal grief and loss due to COVID19 deaths in their family, friend groups, and communities (Sammons et al., 2021). The pandemic may also compound pre-existing race-based stress experienced by psychologists of color, who may personally experience increased racism or responsibilities to support under-resourced communities of color (Miu \& Moore, 2021).

\section{Increased Isolation and Reduced Availability of Consultation}

With these increased stressors on psychologists during the pandemic, peer consultation and support are needed more than ever. Psychologists not only have greater demand and acuity in their clinical practice, but also more isolation (Ledesma \& Fernandez, 2021). Remote work and social distancing have reduced the casual encounters that would have allowed for informal consultation between therapists (Sasangohar et al., 2020). Virtual team meetings are confined to stricter cut-off times to accommodate the next scheduled online meeting or telehealth session. Communication is most often through phone calls and texts, which relays the necessary information but often deprives a space for emotional processing (Sasangohar et al., 2020). Psychologists are missing the "water cooler" and "pop-in" conversations that facilitate informal consultation and clinical reflection among colleagues. These informal conversations open the door to ask quick questions with a fellow psychologist, but they still lack the dedicated time for deeper reflection and support in a peer consultation group.

\section{Assessments and Practical Considerations}

\section{Assessment of Needs for Peer Consultation}

There is no standardized tool to assess the need for peer consultation. It is therefore inherent on psychologists to assess their levels of burnout and self-reflect on their personal and professional struggles. This can indicate potential interference with clinical duties and the importance of peer consultation.

Reliance on subjective evaluations of burnout alone may be inadequate (Carney \& Jefferson, 2014). Psychologist burnout can be best measured by the Maslach Burnout Inventory-Human Services Survey (Maslach et al., 1997). This self-assessment conceptualizes burnout as emotional exhaustion, detachment from patients, and a sense of personal ineffectiveness and lack of accomplishment (Maslach $\&$ Leiter, 2016). Other signs of burnout include negative feelings about clients, reduced quality of care, fatigue, and irritability (Maslach \& Leiter, 2016). In the clinical vignette, Jane would score high on emotional exhaustion as she was "physically and emotionally drained," to the point of being in survival mode trying to get by each day instead of doing her very best. She also endorsed a sense of ineffectiveness and lack of accomplishment when she believed there was minimal progress in therapy. Burnout is particularly common among psychologists who work with challenging populations that do not make immediate treatment gains (Maslach \& Leiter, 2016). This Maslach Burnout Inventory self-assessment can effectively indicate how burnout may interfere with clinical duties and signal the need for peer consultation.

Beyond burnout, psychologists can use the evidencebased Self-Practice/Self-Reflection (SP/SR) program to assess personal and professional challenges (Bennett-Levy et al., 2003). SP/SR engages psychologists to identify beliefs that cause personal and professional struggles. SP/SR increases awareness of clinical effectiveness and addresses unhelpful beliefs that interfere with clinical care. In the clinical vignette, if Jane were to use SP/SR, she could selfreflect on her unrealistic expectation of quickly healing her patients, particularly amid a pandemic. She can reframe her belief by recognizing the role of complex trauma in perpetuating her patients' difficulties. This meta-awareness of her 
self-judgment would help her realize what interferes with her clinical effectiveness.

The Sources of Therapist Stress Questionnaire can further help psychologists identify the root causes that maintain personal and professional challenges (see Bennett-Levy \& Haarhoff, 2019). Based on this questionnaire, Jane would identify the following psychologist stressors: (1) emotional dysregulation and avoidance as client factors, (2) caseload and telehealth navigation as work-related factors, (3) increased childcare responsibilities and the pandemic as event-related factors, (4) negative belief about ability and fear of judgment as self-evaluative factors, and (5) high expectations and taking on complex cases too soon as educational factors. By identifying these therapist stressors, psychologists can more promptly recognize the need for peer consultation.

\section{Effective Peer Consultation Group}

For peer consultation groups to be effective and supportive of clinician needs, groups need to be non-hierarchical, nonevaluative, and non-judgmental, but also honest and confidential (Napan, 2021). These norms create a safe space that allows consultees to be vulnerable about clinical challenges or personal processes, which is especially important for an early career psychologist or new employee as described in the vignette. Successful consultation groups are self-directed and mutually share the task of leadership, where consultees determine their own needs and seek support or resources from the group according to personal needs (Carney \& Jefferson, 2014). Consultation groups demonstrate greater effectiveness when content and process are equally valued, such that the group provides feedback on the clinical case while also exploring the emotional reactions of the consultees (Napan, 2021). This process encourages consultees to openly share mistakes and struggles by normalizing therapists' emotional reactions in addition to clinical challenges. Additionally, successful peer consultation groups adhere to group contract and gatekeeping, manage boundaries, provide constructive feedback, and actively evaluate group functioning and dynamics (Carney \& Jefferson, 2014; Napan, 2021).

Ineffective peer consultation groups can be vulnerable to pitfalls. In a qualitative review of peer consultation groups, Borders (2012) identified that "task drifting" or deviating from pertinent topics can occur in leaderless groups. Groups may not be helpful if members are overly supportive without providing necessary constructive feedback or are too focused on advice giving (Borders, 2012; Napan, 2021). Additionally, groups were less effective when they avoided discussing group dynamics (Borders, 2012). Taken together, effective peer consultation groups can promote clinician wellness and facilitate delivery of efficacious and ethical treatment to patients.

\section{Case Example of a Virtual Peer Consultation Group}

Our peer consultation group was created to address the common clinician needs described in the vignette. We each experienced the impact of the ongoing COVID-19 pandemic on our professional and personal lives in unique ways. Prepandemic, we were accustomed to working in a clinic environment in which we could seek ad hoc consultation and support simply by glancing down the hall and finding the open door of a friendly colleague. Now we experience the professional isolation of remote work, with a loss of spontaneity and need for planned out spaces for professional connection. There was no allocated time and space to consult on challenging cases or process burnout. Simultaneously, there was an increased demand for clinical services and higher acuity in clinical presentations (Sammons et al., 2021).

The group formed following an organic, informal conversation between two of us who connected about feeling isolated clinically and needing a space to discuss patient cases, particularly working with trauma during the pandemic. Two additional psychologists within our department were invited to join, with the vision of creating a small, virtual peer consultation group focused on providing support related to trauma work. We are four psychologists: two early career and two mid-career. One identifies as an Asian American woman, three identify as White women. We all have varying years of practice, years at institution, theoretical orientations, and practice settings. There is a common interest in trauma and social justice. Due to busy caseloads, we decided a monthly, virtual meeting over the lunch hour would best accommodate our schedules without increasing stress.

We were intentional about creating a safe, trusting environment. Because group members had varying levels of relationship history with each other, we took time for personal introductions and group norms. Guided by a relationalcultural framework for supervision and consultation (Jordan, 2004) and effective consultation models (Counselman \& Weber, 2004; Napan, 2021), we started by ascertaining each member's vision of how peer consultation could assist their professional practice. We then agreed on the logistics and structure of our group meetings in keeping with professional guidelines for peer consultation (APA, 2002). We envisioned a space in which we could seek guidance on difficult cases, express our emotional reactions to patients, and provide support around personal circumstances impacting our clinical work. We were intentional about creating a competence constellation (Johnson et al., 2013) where we could bring our authentic selves and facilitate mutual growth, personally and professionally. As such, we decided not to have a specific group leader, with an acknowledgment that any group member could ask for time to discuss concerns. We also agreed to check in periodically about what was working well in our meetings, as well as opportunities for improvement. 
In the many meetings that followed, our consultation group discussed clinical issues related to sexual trauma, grief/loss, life-changing medical diagnoses, and ethical considerations among others. We found great benefit in providing personal support related to a patient death by suicide, experiences of racism, personal challenges during the pandemic, and caregiving stress from children and aging relatives amid increasing work demands. When we introduce our topics during a meeting, we have found added benefit in stating what we look for from our peers: advice, curiosity, community resources, emotional support, etc. There is a general consensus among group members that our peer consultation meetings have helped us process difficult cases and reduce our burnout and professional isolation. We also have expressed feeling less burdened, more supported, and more connected through our common struggles as psychologists during these unprecedented and stressful times.

\section{Lessons Learned}

Based on the current literature (Counselman \& Weber, 2004; Napan, 2021) and our experiences, we provide the following considerations for peer consultation.

1. See consultation as an enriching necessity rather than a luxury or burden. In the face of increased professional challenges and reduced opportunities for informal consultation due to the pandemic and remote work, it is important to be intentional about seeking peer consultation. With a busy clinical schedule, it is easy to overlook or diminish the need for consultation and believe nothing to be urgent enough to interrupt a peer for consultation. However, there is always a need for consultation and it could enrich psychologists' professional perspectives. Consultation encourages psychologists to slow down and reflect on complex cases or professional issues, challenges psychologists to identify their unchecked biases, and counters burnout.

2. Accommodate busy schedule with transition time. Given that psychologists often have back-to-back therapy sessions, it is important to build transition time for consultation meetings. The virtual format reduced the need for transit time. We found it helpful to allow buffer time before and after the next commitment (e.g., end meeting 5 minutes before the next therapy appointment). This transition time allows psychologists to ground themselves in an otherwise busy schedule.

3. Be intentional about choosing the goals, group members, size, and dynamic (Counselman \& Weber, 2004; Napan, 2021). a. Goals: Different psychologists have different needs. It is important to identify fellow consultees who share the same goals. The most common goal is to seek consultation on challenging cases. Our group values forming a connection with a community of psychologists in addition to processing personal and professional issues that could interfere with duties.

b. Group members: It was important to have group members who shared the same professional goals with diverse backgrounds, theoretical orientations, and experiences. This allowed for broad perspectives in case consultation and professional development.

c. Size: We intentionally created a small, closed consultation group so that each consultee could build trust with each other in a community and had adequate space to process individual issues.

d. Dynamic: We found it helpful to have an egalitarian group dynamic, where there is no hierarchy or evaluation of one another. Others may form groups based on learning or clinical needs, such as having a regular case presentation or sharing the same theoretical orientation. However, these groups may not allow for a safe learning environment if a consultee feels judged or evaluated in an open group that does not emphasize non-judgmental norms.

4. Reinforce mutual self-care and self-compassion. An effective psychologist needs to be able to manage personal stressors in order to be present for patients' struggles. A peer consultation group can check in with one another compassionately and non-judgmentally. With each other's encouragement of self-care and selfcompassion, psychologists will be reminded to prioritize self-care (e.g., sleep, healthy diet, and exercise). The group can also be compassionate to each other, as psychologists are also humans who make mistakes and struggle.

\section{Key Clinical Considerations}

- See peer consultation as an enriching necessity rather than a luxury or burden.

- Accommodate busy schedules with transition time before next meeting.

- Choose an egalitarian, small, closed, peer consultation group with shared goals of forming a community of trust and support.

- Reinforce mutual self-care and compassion in the peer consultation group. 


\section{Conclusion}

The pandemic imposed additional burdens on psychologists while reducing their support. Peer consultation is now an enriching necessity rather than a luxury. Remote or hybrid work for psychologists is here to stay beyond the pandemic, which calls for ongoing solutions that reduce clinician isolation and burnout. Together, in a competence constellation, peer consultees can form a community that advances each other's well-being and professional competence. With a nonjudgmental, compassionate consultation group, psychologists can better sustain challenging yet rewarding clinical work.

\section{Declarations}

Disclosures The authors have no conflict of interests or disclosures.

\section{References}

Aafjes-van Doorn, K., Békés, V., Prout, T. A., \& Hoffman, L. (2020). Psychotherapists' vicarious traumatization during the COVID-19 pandemic. https://doi.org/10.1037/tra0000868

American Psychological Association (2002). Ethical principles of psychologists and code of conduct. American Psychologist, 57(12), 1060-1073. https://doi.org/10.1037/0003-066X.57.12.1060

Bennett-Levy, J. \& Haarhoff, B. (2019). Why therapists need to take a good look at themselves. In S. Dimidjian (Eds), Evidence-based practice in action: Bridging clinical science and intervention (pp.380-394). Guilford Press.

Bennett-Levy, J., Lee, N., Travers, K., Pohlman, S., \& Hamernik, E. (2003). Cognitive therapy from the inside: Enhancing therapist skills through practising what we preach. Behavioural and Cognitive Psychotherapy, 31(2), 143-158. https://doi.org/10.1017/S1352 465803002029

Borders, L. D. (2012). Dyadic, triadic, and group models of peer supervision/consultation: What are their components, and is there evidence of their effectiveness?. Clinical Psychologist, 16(2), 59-71. https://doi.org/10.1111/j.1742-9552.2012.00046.x

Carney, J \& Jefferson, J. (2014). Consultation for mental health counselors: Opportunities and guidelines for private practice. Journal of Mental Health Counseling, 36 (4), 302-314. 10.17744/ mehc.36.4.821133r0414u37v7

Counselman, E. F., \& Weber, R. L. (2004). Organizing and maintaining peer supervision groups. International Journal of Group Psychotherapy, 54(2), 125-143. https://doi.org/10.1521/ijgp.54.2.125. 40391

Isobel R.B, Bell, K., Kalle, W., Pawar, M. (2014). Restoring meaning to supervision through a peer consultation group in rural Australia. Journal of Social Work Practice, 28(4), 479-495. https:// doi.org/10.1080/02650533.2014.896785

Johnson, W. B., Barnett, J. E., Elman, N. S., Forrest, L., \& Kaslow, N. J. (2013). The competence constellation model: A communitarian approach to support professional competence. Professional Psychology: Research and Practice, 44(5), 343-354. https://doi.org/10.1037/a0033131

Jordan, J. V. (2004). Relational learning in psychotherapy consultation and supervision. In Walker \& Rosen (Eds), How
Connections Heal: Stories from Relational-Cultural Therapy (pp. 22-27). New York, NY: Guilford Publications.

Ledesma, D. A. S., \& Fernandez, K. T. G. (2021). 'If I am not well, I can't do sessions well': An analysis of the narratives of Filipino Therapists during the COVID-19 Pandemic. Counselling and Psychotherapy Research, 1-10. https://doi.org/10.1002/capr. 12442

Lewis, G. J., Greenburg, S. L., \& Hatch, D. B. (1988). Peer consultation groups for psychologists in private practice: A national survey. Professional Psychology: Research and Practice, 19(1), 81-86. https://doi.org/10.1037/0735-7028.19.1.81

Maslach, C., Jackson, S. E., \& Leiter, M. P. (1997). Maslach Burnout Inventory: Third edition. In C. P. Zalaquett \& R. J. Wood (Eds.), Evaluating stress: A book of resources (pp. 191-218). Scarecrow Education.

Maslach, C. \& Leiter, M. P. (2016). Understanding the burnout experience: Recent research and its implications for psychiatry. World Psychiatry, 15(2), 103-111. https://doi.org/10.1002/wps.20311

Miu, A. S. \& Moore, J. R. (2021). Behind the masks: Experiences of mental health practitioners of color during the COVID-19 pandemic. Academic Psychiatry, 1-6. https://doi.org/10.1007/ s40596-021-01427-w

Miu, A. S., Vo, H. T., Palka, J. M., Glowacki, C. R., \& Robinson, R. J. (2020). Teletherapy with serious mental illness populations during COVID-19: Telehealth conversion and engagement. Counselling Psychology Quarterly, 1-18. https://doi.org/10.1080/09515070. 2020.1791800

Napan, K. (2021). The spirit of peer supervision. In K. O'Donoghue \& L. Engelbrecht (Eds.), The Routledge international handbook of social work supervision. Routledge.

Panchal, N., Kamal, R., Cox, C., \& Garfield, R. (2021, February 10). The implications of COVID-19 for mental health and substance use. Kaiser Family Foundation. https://www.kff.org/coronaviruscovid-19/issue-brief/the-implications-of-covid-19-for-mentalhealth-and-substance-use/

Sammons, M. T., Elchert, D. M., \& Martin, J. N. (2021). Mental health service provision during COVID-19: Results of a third survey of licensed psychologists. Journal of Health Service Psychology, 47, 119-127. https://doi.org/10.1007/s42843-021-00044-3

Sasangohar, F., Bradshaw, M. R., Carlson, M. M., Flack, J. N., Fowler, J. C., Freeland, D., ... \& Madan, A. (2020). Adapting an outpatient psychiatric clinic to telehealth during the COVID-19 pandemic: A practice perspective. Journal of Medical Internet Research, 22(10), e22523. https://doi.org/10.2196/22523

Shklarski, L., Abrams, A., \& Bakst, E. (2021). Navigating changes in the physical and psychological spaces of psychotherapists during Covid-19: When home becomes the office. Practice Innovations, 6(1), 55-66. https://doi.org/10.1037/pri0000138

Slone, H., Gutierrez, A., Lutzky, C., Zhu, D., Hedriana, H., Barrera, J. F., ... \& Bunnell, B. E. (2021). Assessing the Impact of COVID19 on Mental Health Providers in the Southeastern United States. Psychiatry Research, 302, 114055. https://doi.org/10.1016/j.psych res.2021.114055

Stewart, R. E., \& Chambless, D. L. (2008). Treatment failures in private practice: How do psychologists proceed? Professional Psychology: Research and Practice, 39(2), 176-181. https://doi.org/ 10.1037/0735-7028.39.2.176

Stoll, J., Müller, J. A., \& Trachsel, M. (2020). Ethical issues in online psychotherapy: A narrative review. Frontiers in Psychiatry, 10(993), 1-16. https://doi.org/10.3389/fpsyt.2019.00993

Publisher's Note Springer Nature remains neutral with regard to jurisdictional claims in published maps and institutional affiliations. 
Adriana S. Miu, PhD, ABPP, is an Assistant Professor in the Department of Psychiatry at the University of Texas Southwestern Medical Center. She is a licensed psychologist since 2017 and received board certification in clinical psychology in 2021. She works at an adult outpatient clinic, with specializations in sexual trauma and early psychosis. Her research interests include social cognition, growth mindset, and resilience.

Anitha Joseph, PhD, is an Assistant Professor in the Department of Psychiatry at the University of Texas Southwestern Medical Center. She is a licensed psychologist since 2012. She provides clinical care in an adult outpatient clinic with a specialization in anxiety and obsessivecompulsive disorder treatment and transition age youth population.

Ellie Hakim, PhD, is an Assistant Professor in the Department of Psychiatry at the University of Texas Southwestern Medical Center. She is a licensed psychologist since 2004. She provides clinical services to graduate and medical students in the Student Wellness and Counseling Center. Her professional interests include supervision, graduate psychology training, identity development, and family of origin issues.
Erin D. Cox, PsyD, is an Assistant Professor at the University of Texas Southwestern Medical Center. Licensed as a psychologist since 2013, she provides care to undergraduate and graduate students in university counseling center settings with a specialization in eating disorder treatment.

Ellen Greenwald, $\mathbf{P h D}$, is an Assistant Professor in the Department of Psychiatry at the University of Texas Southwestern Medical Center. She is a licensed psychologist since 2010 and her clinical role is with the Resident Wellness and Counseling Center. She teaches doctoral students in clinical psychology, and her professional foci are diversity/ social justice advocacy, trauma recovery, mindfulness, sexuality, and graduate psychology training. 